APS/123-QED

\title{
Evolution of massive fields around a black hole in Hořava gravity
}

\author{
Nijo Varghese and V C Kuriakose* \\ Department of Physics, Cochin University of Science and Technology, \\ Cochin - 682 022, Kerala, India.
}

(Dated: June 14, 2018)

\begin{abstract}
We study the evolution of massive scalar field in the spacetime geometry of Kehagias-Sfetsos(KS) black hole in deformed Hořava-Lifshitz(HL) gravity by numerical analysis. We find that the signature of HL theory is encoded in the quasinormal mode(QNM) phase of the evolution of field. The QNM phase in the evolution process lasts for a longer time in HL theory. QNMs involved in the evolution of massive field are calculated and find that they have a higher oscillation frequency and a lower damping rate than the Schwarzschild spacetime case. We also study the relaxation of field in the intermediate and asymptotic range and verified that behaviors of field in these phases are independent of the HL parameter and is identical to the Schwarzschild case.
\end{abstract}

*Electronic address: nijovarghese@cusat.ac.in, vck@cusat.ac.in 


\section{INTRODUCTION}

One of the major open challenges facing theoretical physics is to accommodate general relativity(GR) in the framework of quantum field theory. The main problem one encounters is the non-renormalizablity of gravity at the perturbative level. Hořava[1] suggested a powercounting renormalizable theory of gravity in $3+1$ dimensions inspired from the Lifshitz model now dubbed as Horava-Lifshitz gravity. It assumes a Lifshitz-like anisotropic scaling between space and time at short distances, characterized by a dynamical critical exponent $\mathrm{z}=3$ and thus breaking the Lorentz invariance. While in the IR limit it flows to $\mathrm{z}=1$, retrieving the Einstein's GR. Even though many of the fundamental questions have not yet been answered[2, 3] the seemingly innovative ideas of HL theory ignited an interest in the topic and a number of works has been reported based on it. Since the theory has the same Newtonian and post Newtonian corrections as those of GR, systems of strong gravity, like black holes, are needed to get observable deviation from the standard GR. Various black hole solutions [4 23] are found in HL theory of which one with asymptotically flat Minkowski spacetime is the KS black hole given in[24] by applying deformation in the original theory. Various aspects of KS black hole were explored in the past [25 37].

The study of response of the black hole to external perturbations is the best way to get a clear picture of the properties of these exotic objects. As it is well established, the evolution of perturbations in black hole spacetimes involves three stages[46]. The first one is an initial response containing the information of the particular form of the original wave field followed by a region dominated by damped oscillation of the field called quasinormal modes, which depends entirely on the background black hole spacetimes. The QNMs play an important role in almost all astrophysical processes involving black holes and the spectra of QNMs may be different in various theories of gravity and would help us to distinguish these theories. The late time tail stage dominates in the final phase of evolution.

Evolution of different fields and the associated QNMs were studied in HL black holes in the frequency domain [38 43] and in time domain[44]. All these studies on the field evolution around black holes in HL theory has so far been restricted to the massless fields. Evolution of massive field is a more intriguing subject of study. The spectrum of entropy/area is discussed in the viewpoint of QNMs of massive scalar field in[45]. It is well understood in standard GR that massive field behaves differently from the massless one. It was confirmed 
that the massive fields decay more slowly than massless one[49]. The late-time behavior of the field that follows the QNM stage of evolution is also an interesting topic of study since it reveals the actual physical mechanism by which a perturbed black hole sheds its hairs. It was first demonstrated by Price[61] that in the background of the Schwarzschild spacetime the massless neutral field at late time dies off as $\Psi \sim t^{-(2 \ell+2)}$ or $\Psi \sim t^{-(2 \ell+3)}$ depending on the initial conditions and the multipole order $\ell$. In contrast with the massless fields, massive fields have an oscillatory inverse power-law behavior, with $\Psi \sim t^{-(\ell+3 / 2)} \sin (m t)$ at intermediate late times $M m \ll m t \ll 1 /(m M)^{2}[48]$. But it was shown analytically that in the asymptotic late times $\left(m t \gg 1 /(m M)^{2}\right)$ another pattern of oscillatory tail of the form $\Psi \sim t^{-(5 / 6)} \sin (m t)$ dominates[50] and it was numerically verified for various space times and fields [52 55]. All these studies argued that late time relaxation does not have any relation to the space time parameters. Inspired from the hints that QNMs show dependence on spacetime parameter in three dimensional AdS[56] and dS[57] space times, a detailed numerical study of relaxation process in $\mathrm{RN}$ space time was done in [58]. They showed that for $M m \ll 1$ relaxation depends only on the field parameters, but when $M m \gg 1$ spacetime parameters affect the relaxation and found that for a Schwarzschild black hole bigger the black hole mass is, the faster the perturbation decays. The purpose of this paper is to investigate the different stages of evolution of a massive scalar field around black hole in HL theory.

The paper is organized as follows. In Sec II we derive the wave equation for massive scalar field around KS black hole and the numerical method employed to study the time evolution is explained and in Sec.III the evolution of a massless field is studied using the method of time domain integration. The massive field perturbation is studied in Sec [V] and the results are summarized in Sec.V.

\section{SCALAR FIELD AROUND KS BLACK HOLE}

In [24] Kehagias and Sfetsos obtained a spherically symmetric black hole solution by deforming HL gravity that allows asymptotically flat spacetimes with the addition of a term proportional to the Ricci scalar of three-geometry, $\mu^{4} R^{(3)}$, with the cosmological constant $\Lambda_{W} \rightarrow 0$. This will not alter the UV properties of the theory but it does the IR ones leading to Minkowski vacuum analogous to Schwarzschild spacetime in GR. The external 
gravitational field of such a black hole has the form [24],

$$
d s^{2}=-N(r)^{2} d t^{2}+f(r)^{-1} d r^{2}+r^{2} d \Omega^{2},
$$

where $f(r)$ has the form,

$$
N(r)^{2}=f(r)=\frac{2\left(r^{2}-2 M r+\alpha\right)}{r^{2}+2 \alpha+\sqrt{r^{4}+8 \alpha M r}} .
$$

The event horizons are at,

$$
r_{ \pm}=M \pm \sqrt{M^{2}-\alpha}
$$

When the HL parameter $\alpha=0$ the solution reduces to the Schwarzschild spacetime. We will now consider the evolution of scalar field in this spacetime. Scalar field evolution is governed by the Klein-Gordon equation,

$$
\frac{1}{\sqrt{-g}} \partial_{\mu}\left(\sqrt{-g} g^{\mu \nu} \partial_{\nu}\right) \Phi-m^{2} \Phi=0
$$

Resolving the scalar field into spherical harmonics, $\Phi(t, r, \theta, \phi)=\frac{1}{r} \Psi_{\ell}(t, r) Y_{\ell m}(\theta, \phi)$ and employing the tortoise coordinate defined by $d r_{*}=\frac{1}{f} d r$, the above equation can be reduced to the form,

$$
\left(-\frac{\partial^{2}}{\partial t^{2}}+\frac{\partial^{2}}{\partial r_{*}^{2}}\right) \Psi_{\ell}(t, r)=-V_{\ell}(r) \Psi_{\ell}(t, r)=0,
$$

where the effective potential $V_{\ell}(r)$ is given by

$$
V_{\ell}(r)=f(r)\left[\frac{\ell(\ell+1)}{r^{2}}+\frac{1}{r} \frac{\partial f(r)}{\partial r}+m^{2}\right]
$$

The behavior of the effective potential $V_{\ell}(r)$ is plotted in Fig 1. In Fig:1(a) the dependence of the potential with $\alpha$ is shown for massless and massive $(m=0.3)$ fields. For a given mass, as the parameter $\alpha$ increases the peak of the potential increases where as the asymptotic region of potential is unaffected. It is clear from Fig.1(b) that as the mass of the field increases the hight of the potential increases and its asymptotic value raises as $m^{2}$. The barrier nature of potential will be spoiled for high field masses.

The complex nature of the potential makes it difficult to obtain the exact solutions of $\mathrm{Eq}(15)$ and we have to tackle the problem by numerical methods. The wave equation Eq(15) can be recasted in the null coordinates $u=t-r^{*}$ and $v=t+r^{*}$ as,

$$
-4 \frac{\partial^{2}}{\partial u \partial v} \Psi(u, v)=V(u, v) \Psi(u, v) .
$$



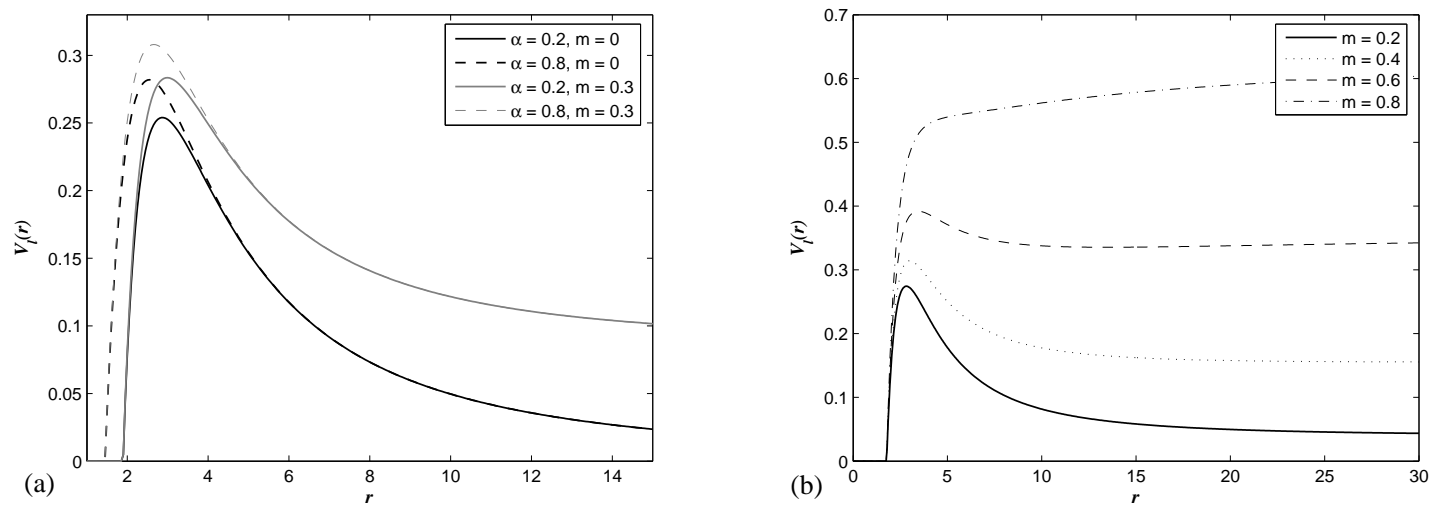

FIG. 1: Profiles of effective potential $V_{\ell}(r)$ for $\ell=2$. (a) $V_{\ell}(r)$ for $\alpha=0.2$ (solid) and $\alpha=$ 0.8 (dotted). (b) $V_{\ell}(r)$ with different field masses for $\alpha=0.4$.

The above equation is invariant under the resealing

$$
r \rightarrow a r, \quad t \rightarrow a t, \quad M \rightarrow a M, \quad u \rightarrow u / a, \quad \alpha \rightarrow a^{2} \alpha
$$

for some positive constant $a$, such that $M m$ is invariant. We numerically integrate this equation using the difference scheme[59],

$$
\Psi_{N}=\Psi_{W}+\Psi_{E}-\Psi_{S}-\frac{h^{2}}{8} V(S)\left(\Psi_{W}+\Psi_{E}\right)+O\left(h^{4}\right)
$$

The integration is performed on an uniformly spaced grid with points $N(u+h, v+h)$, $W(u+h, v), E(u, v+h)$ and $S(u, v)$ forming a null rectangle with an overall grid scale factor of $h$. Since the late-time behavior of the wave function is found to be insensitive to the initial data, we set $\psi(u, v=0)=0$ and a Gaussian profile $\Psi(u=0, v)=\operatorname{Aexp}\left[-\frac{\left(v-v_{0}\right)^{2}}{2 \sigma^{2}}\right]$. In all our calculations we set the initial Gaussian with width $\sigma=3$ centered at $v_{0}=10$. Due to the linearity of equation one has the freedom to choose the amplitude of the initial wave $A=1$. To proceed the integration on the above numerical scheme one has to find the value of the potential at $r\left(r_{*}\right)=r((v-u) / 2)$ at each step. For this, we numerically integrate the equation for the tortoise coordinate using the Runge-Kutta method[60] and by cubic spline interpolation, obtained $r\left(r_{*}\right)$ at each step. 


\section{EVOLUTION OF MASSLESS FIELD}

Before studying the massive case, we first examine the massless scalar field $(m=0)$ evolution around KS black hole. In Fig 2 (a) time evolution of a typical $\ell=2$ field in HL theory is compared with the corresponding Schwarzschild case. We can see that the QNM phase extends for a longer time in HL theory. The late-time tail starts at a later time $t \approx 415$ for KS black hole with $\alpha=0.8$ whereas it is at $t \approx 320$ for the Schwarzschild case. It is also clear from the figure that the oscillation frequency and the damping time have a higher values in HL theory.

After the QNM stage the massless field dies off as an inverse power of time $\Psi \sim t^{-(2 \ell+3)}$. Fig_2(b) shows the late-time behavior of wave function for different values of $\alpha$, with multipole index $\ell=2$. It is clear from the figure that the late-time behavior massless field decay is independent of $\alpha$ and is same as for the Schwarzschild black hole.
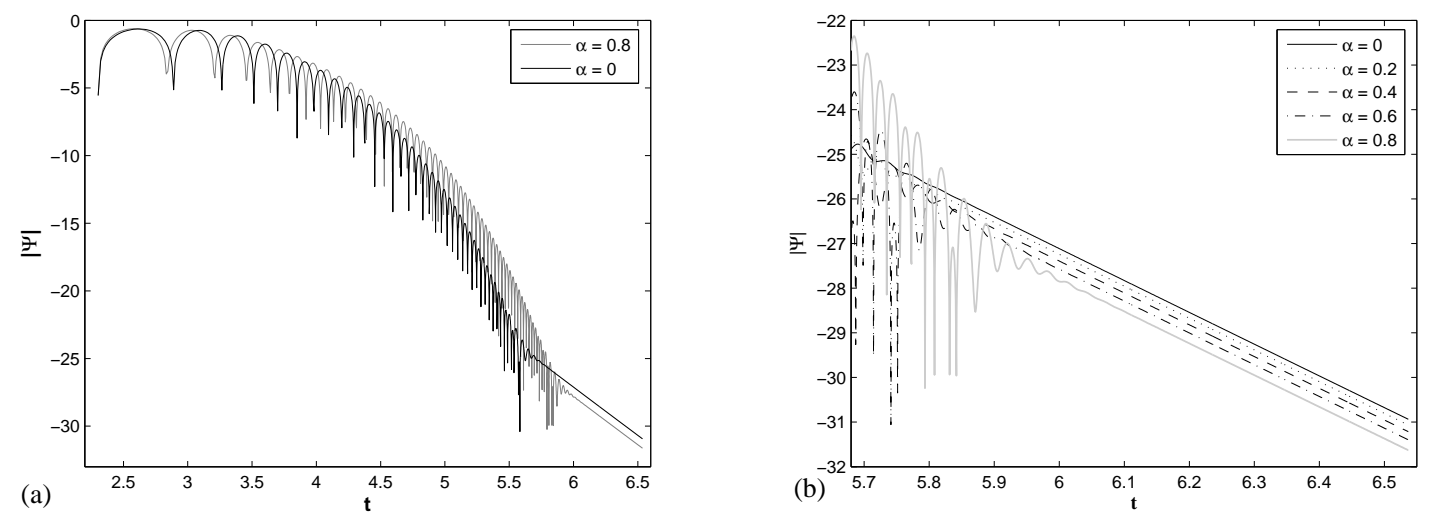

FIG. 2: Temporal evolution of massless scalar field in log-log scale with $\ell=2$. (a) Wave function in KS spacetime with $\alpha=0.8$ in comparison with that in $\operatorname{Schwarzschild~spacetime}(\alpha=0)$. (b)Late time behavior of the field for deferent values of $\alpha$.

In Fig, 3, field evolution for different multipole indices are explored for $\alpha=0.4$. The field falls off as $\psi \sim t^{-3.14}, t^{-5.13}, t^{-7.12}$ and $t^{-9.13}$ for $\ell=0,1,2$ and 3 respectively with the predicted powers $-3,-5,-7$ and -9 respectively. 

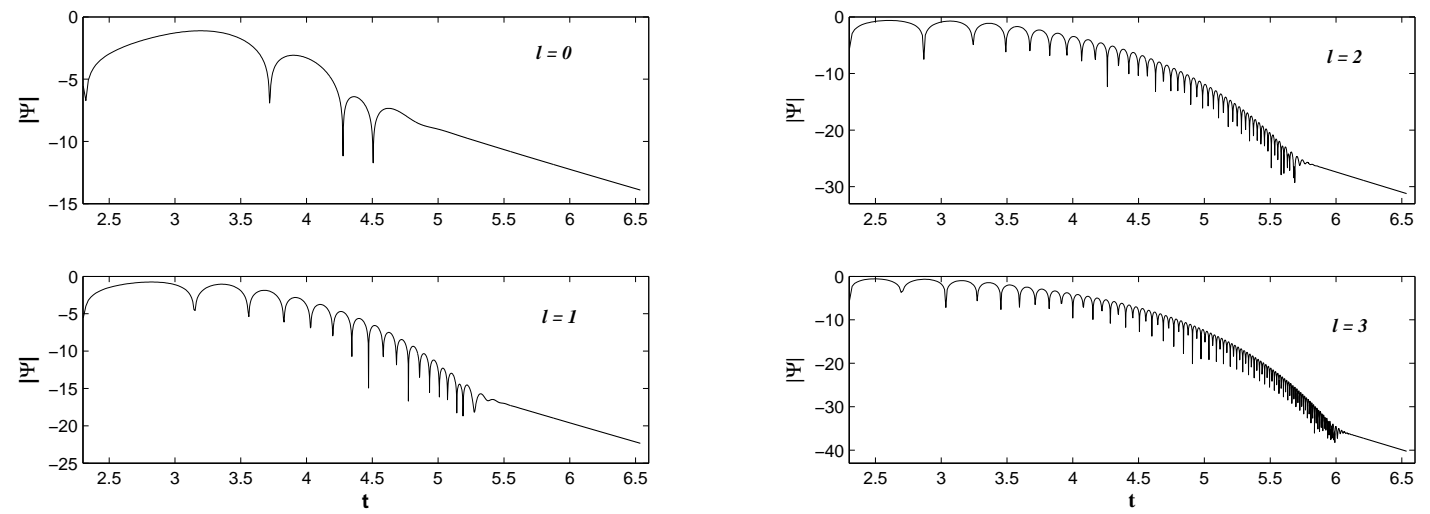

FIG. 3: Decay of field having different $\ell$. The late-time tail dies off with power-law exponents $-3.14,-5.13,-7.12$ and -9.13 for $\ell=0,1,2$ and 4 respectively.

\section{EVOLUTION OF MASSIVE FIELD}

In this section we consider the massive field evolution in the spacetime of KS black hole. We will compare the results with the existing results in standard GR. Here in the first series of experiments we set the mass of the black hole, $M=1$ and choose the the field mass $m$ such that $M m \leq 1$. In Fig 4(a)the wave function for massive scalar field $(m=0.1)$ is plotted in comparison with the corresponding Schwarzschild case for $\ell=2$. After the prompt response in the beginning the quasinormal ringing starts. We can see a clear difference between the two cases in the QNM region that the QNM phase ends at a later time in HL theory and has a lower damping rate. Fig 4(b) shows the variation of QNM with the field mass $m$ for $\alpha=0.4$. As the mass of the field increases the QNM phase shrinks in time.

To see the effects of the HL parameter $\alpha$ and field mass $m$ in QNM phase, we calculate the exact values of QNMs from the numerically integrated data in the time domain by a nonlinear $\chi^{2}$ fitting. We also use the third order WKB method [63], an efficient semianalytic method originally developed by Schutz and Will[62] for the lowest order. The method is found to be accurate for low lying modes and can be used to explore the QNM behavior of black holes for field with very low mass. The calculated values are given in Table【and Table II. QNMs in HL theory have a higher oscillation frequency $R e(\omega)$ and a lower damping rate $|\operatorname{Im}(\omega)|$ than the Schwarzschild spacetime case. Also the $\operatorname{Re}(\omega)$ and $|\operatorname{Im}(\omega)|$ found to be decreasing with the increase of the HL parameter $\alpha$. WKB method gives verse results for higher field masses since the barrier nature of potential gets spoiled at these mass ranges. 

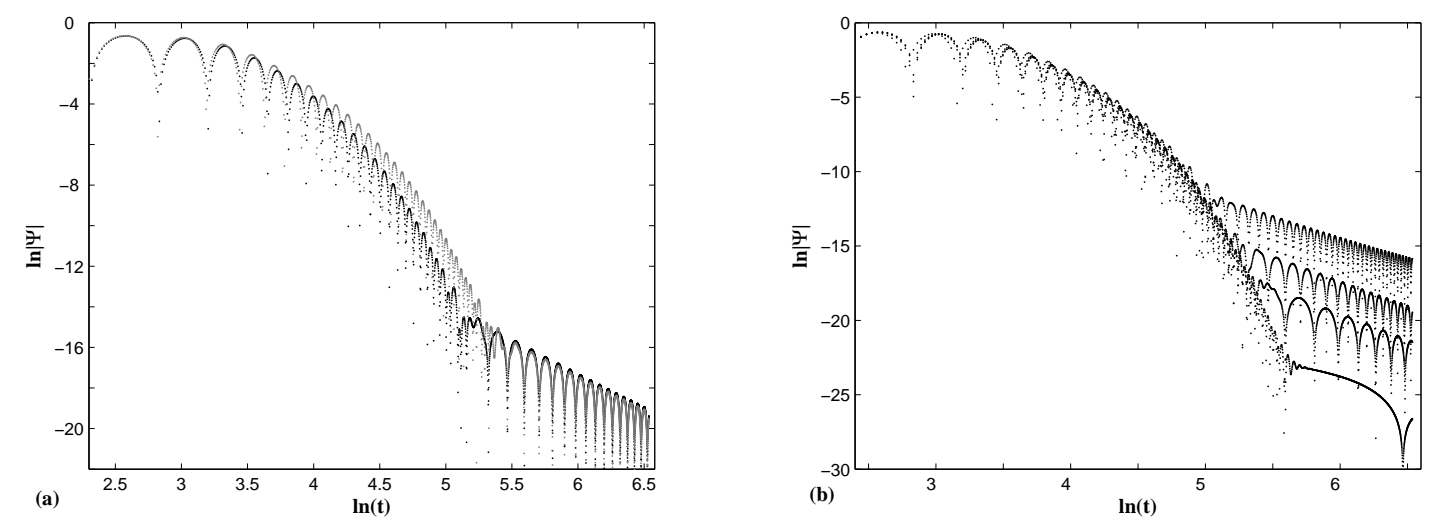

FIG. 4: Time evolution of massive scalar field for $\ell=2$ mode. (a)Field of mass, $m=0.1$ in KS spacetime with $\alpha=0.8$ (top curve) in comparison with the corresponding case in Schwarzschild spacetime(bottom curve). (b)Field with different masses for $\alpha=0.4$, curves from bottom to top is for $m=0.01,0.05,0.1$ and 0.2 .

So there should be a discrepancy between the QNMs evaluated by time domain and WKB methods at high field mass.

\begin{tabular}{llc|cc}
\hline \hline & \multicolumn{2}{c|}{ WKB } & \multicolumn{2}{c}{ Time domain } \\
$\alpha$ & $\operatorname{Re}(\omega)$ & $\operatorname{Im}(\omega)$ & $\operatorname{Re}(\omega)$ & $\operatorname{Im}(\omega)$ \\
\hline 0 & 0.48637 & -0.09572 & 0.48552 & -0.09573 \\
0.2 & 0.49388 & -0.09253 & 0.49377 & -0.09256 \\
0.4 & 0.50231 & -0.08884 & 0.50159 & -0.08888 \\
0.6 & 0.51188 & -0.08438 & 0.51142 & -0.08437 \\
0.8 & 0.52293 & -0.07868 & 0.52143 & -0.07893 \\
1 & 0.53587 & -0.07069 & 0.53569 & -0.07106 \\
\hline \hline
\end{tabular}

TABLE I: Fundamental $(n=0)$ QNM frequencies of massive $(m=0.1)$ scalar field for $\ell=2$, calculated using WKB and numerical integration data

The QNM phase is followed by the phase of late time tail behavior of the field decay. First we consider the late time tails in intermediate range, $M m \ll m t \ll(m M)^{2}$. The decay of field along black hole outer horizon $H_{+}$(approximated by the null surface $u_{\max }=0.5 \times 10^{4}$ ) and future time like infinity $i_{+}$(approximated on fixed radius $r_{*}=50 M$ ) are evaluated with 


\begin{tabular}{lll|cc}
\hline \hline & \multicolumn{2}{c|}{ WKB } & \multicolumn{2}{c}{ Time domain } \\
$m$ & $\operatorname{Re}(\omega)$ & $\operatorname{Im}(\omega)$ & $\operatorname{Re}(\omega)$ & $\operatorname{Im}(\omega)$ \\
\hline 0 & 0.49942 & -0.08969 & 0.49886 & -0.08951 \\
0.02 & 0.49953 & -0.08966 & 0.49979 & -0.08969 \\
0.05 & 0.50014 & -0.08948 & 0.49839 & -0.08948 \\
0.07 & 0.50083 & -0.08928 & 0.50156 & -0.08922 \\
0.1 & 0.50231 & -0.08883 & 0.50159 & -0.08875 \\
0.2 & 0.51101 & -0.08622 & 0.51083 & -0.08701 \\
0.3 & 0.52571 & -0.08169 & 0.52737 & -0.08029 \\
0.4 & 0.54665 & -0.07501 & 0.53933 & -0.06758 \\
0.5 & 0.57423 & -0.06568 & 0.54165 & -0.05451 \\
\hline \hline
\end{tabular}

TABLE II: QNM frequencies afor different mass of the scalar field $m$. QNMs calculated using WKB and numerical integration data $(\ell=2$ and $\alpha=0.4)$

the initial field profile parameters $v_{0}=50$ and $\sigma^{2}=2$. The result for $\alpha=0.4$ with $m=0.01$ and $\ell=0$ is shown in Fig 5 (a). After the QNM phase the amplitude of the oscillatory field decays as a power law with exponent -1.49 along $I_{+}$and $H_{+}$with a period of oscillation $T=314.8$. We have analyzed for different values of HL parameter $\alpha$ and found that the tail behavior is almost independent of $\alpha$ and the decay is identical to the Schwarzschild case according to the form,

$$
\Psi \sim t^{-(\ell+3 / 2)} \sin (m t)
$$

This can be expected since the late-time tail is originated by the backscattering by the effective potential in the asymptotic region and as it is clear from Fig.1, that the parameter $\alpha$ has no effect on this asymptotic region of the potential but can only change its shape near the peek.

The field decay for different multipole indices is plotted in Fig.5.(b) with $m=0.01$. The decaying tail is found to have a period of oscillations $T=314.7 \pm 0.03$ and the power-law exponents $-1.49,-2.5,-3.51$ and -4.51 for $\ell=0,1,2$, and 3 respectively. An excellent agrement with the decay rate of the form $t^{-(\ell+3 / 2)}$ can be seen. We find that the frequency 

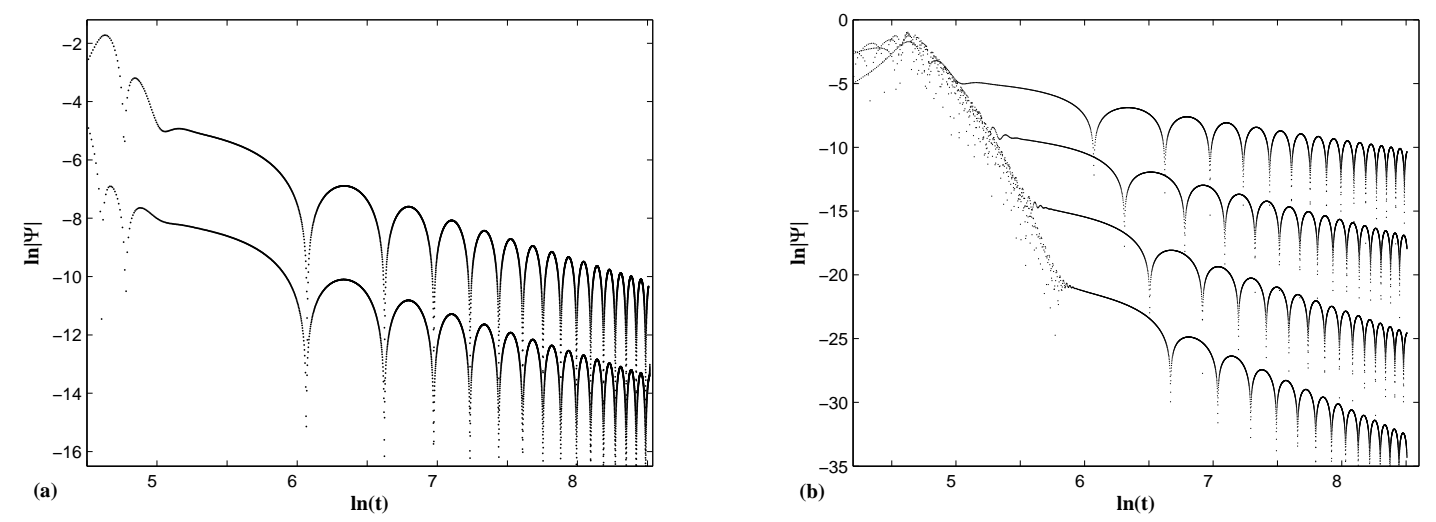

FIG. 5: Decay of massive field with $m=0.01$. (a) Field along outer horizon $H_{+}$(top curve) and future time like infinity $i_{+}$(bottom curve). The amplitude of field decay as power law with exponent -1.49 and period of oscillation $T=314.8(b)$ Field along $i_{+}$for different $\ell$. The field dies off as power-law with exponents $-1.49,-2.5,-3.51$ and -4.51 for $\ell=0,1,2$, and 3 respectively. The period of oscillation $T=314.7 \pm 0.03$.

and damping rate of the oscillatory tails are independent of the parameter $\alpha$ and follow the Schwarzschild case. Thus Eq(10) will be the form of the intermediate late-time behavior of massive field decay in HL theory also.
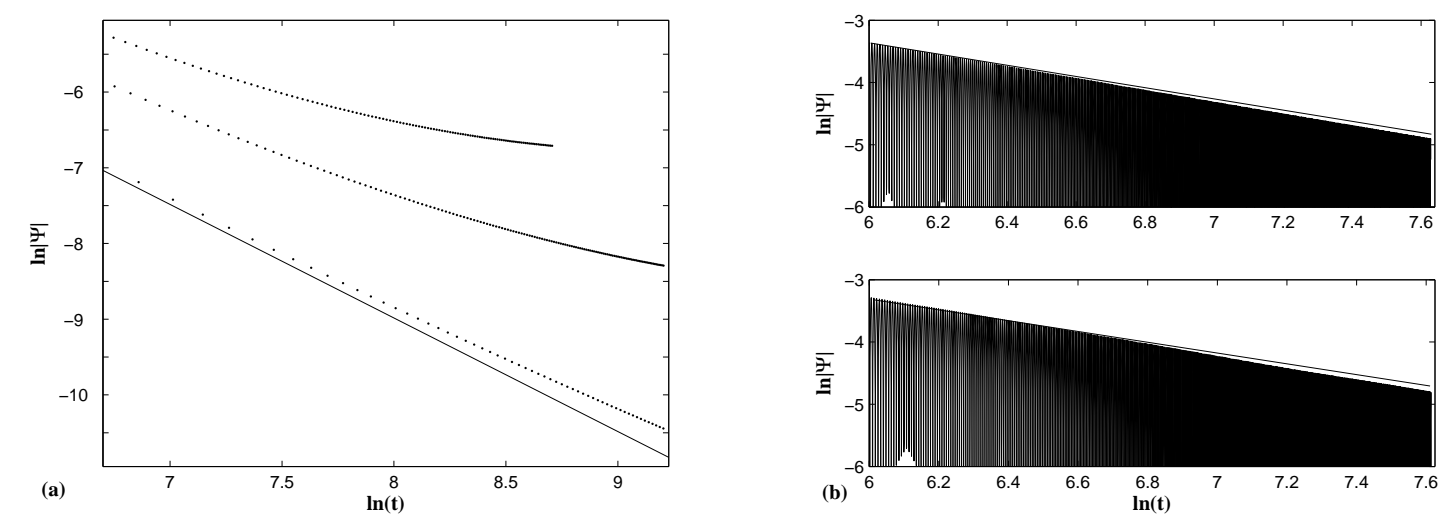

FIG. 6: Late time behavior of field for $\alpha=0.4$. (a)Maxima of oscillation for $\ell=0$, from bottom to top $m=0.02,0.05,0.07$. Solid line has a slop -1.5 . (b)Asymptotic regime of field decay with $m=1$ for $\ell=0$ (top) and $\ell=2$ (bottom). Straight lines have a slop $-5 / 6$.

But in the asymptotic late time the decay of field does not follow pattern given by Eq(10). Another pattern of oscillatory tail dominates in the asymptotic late times $m t \gg 1 /(M m)^{2}$. 
This can be seen from Fig 5 (a), where the maxima of oscillations are shown for $\alpha=0.4, \ell=0$ and $m=0.02,0.05,0.07$. The deviation from straight line with slop -1.5 is visible in the asymptotic late time. When $m t \gg 1 /(M m)^{2}$ smaller the $M m$ the later the asymptotic tail starts. In Fig 5 (b) asymptotic region is shown for $m=1$ and $\ell=0$ and 2 . We find that the asymptotic tail is independent of the multipole order and follows the $\Psi \sim t^{-5 / 6} \sin (m t)$ form as in the standard GR. We have analyzed the asymptotic tail for different values of $\alpha$ and find that this regime is also independent of the HL parameter $\alpha$.

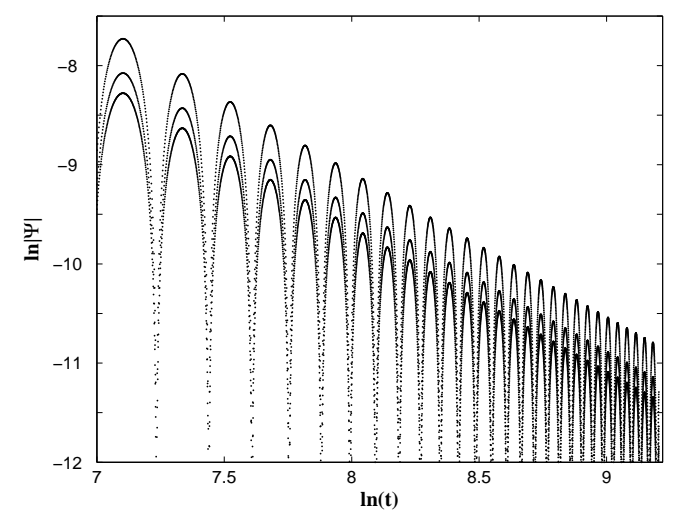

FIG. 7: Decay of field with different black hole mass for $m=0.01, \alpha=0.4$ and $\ell=0$. For $M=0.5,1$ and 1.5 (curves from top to bottom) the decay rates of field are $-1.458,-1.459$ and -1.462 respectively.

Now we study the role of the black hole parameter on the decay of field for $M m \ll 1$. The numerical results for different black hole mass with field mass $m=0.01$ are shown in Fig.7. The field falls off with power law exponents $-1.458,-1.459$ and $M=0.5,1$ and 1.5 respectively and we can conclude that for $M m \ll 1$ the decay rate is independent of black hole mass. We are not going for the $M m \gg 1$ case of computationally expensive part of study. For $M m \gg 1$ range we have good reasons to believe that the relaxation will show similar results of the Schwarzschild case as shown in [58], since we have already shown that the parameter $\alpha$ has no effect on the asymptotic region of the potential and the late time relaxation is independent of the HL parameter. 


\section{SUMMARY}

We have studied the time evolution of massive scalar field in the spacetime geometry of KS black hole in deformed HL gravity by time domain integration. We find a noticeable deviation of the evolution behavior of massive field in the ringdown region from the standard Schwarzschild black hole. QNMs involved in the evolution of massive field are calculated from the time domain integration data and third order WKB method. Massive QNMs in HL theory have a higher oscillation frequency and a lower damping rate than the Schwarzschild spacetime case. The ringdown frequency and damping rate also decrease with the increase of the HL parameter $\alpha$. As the field mass increases the QNM phase squeezes to a smaller time interval. However the late time evolution of massive field fails to show any distinction

from the Schwarzschild case. In the intermediate range the field decays as $t^{-(\ell+3 / 2)} \sin (m t)$, but in the asymptotic late time the decay is dominated by $t^{-5 / 6} \sin (m t)$ tail. In the range $m t \gg 1 /(M m)^{2}$, smaller the value $M m$ the later the asymptotic tail starts. We also shown that for $M m \ll 1$ the relaxation is independent of space time parameters. Since the HL parameter $\alpha$ has no effect on the asymptotic region of the potential the late time relaxation is independent of the HL parameter.

\section{Acknowledgements}

NV wishes to thank UGC, New Delhi for financial support under RFSMS scheme. VCK is thankful to CSIR, New Delhi for financial support under Emeritus Scientistship scheme and wishes to acknowledge Associateship of IUCAA, Pune, India.

[1] P. Hořava, Phys. Rev. D. 79 084008(2009), P. Hořava, JHEP 03 020(2009), P Hořava, Phys. Rev. Lett. 102 161301(2009).

[2] A Padilla, J. Phys. Conf. Ser. 259 012033(2010).

[3] T. P. Sotiriou, J. Phys. Conf. Ser. 283 012034(2011).

[4] H. Lu, J Mei and C. N. Pope, Phys. Rev. Lett. 103 091301(2009).

[5] Mu-In Park, it JHEP 09 123(2009).

[6] H. Nastase, arXiv:0904.3604 [hep-th]. 
[7] R. G. Cai, Y. Liu and Y. Sun , JHEP 06 010(2009).

[8] R. G. Cai, L. Cao and N. Ohta, Phys. Rev. D $80024003(2009)$.

[9] A. Ghodsi and E. Hatefi, Phys. Rev. D 81 044016(2010).

[10] H. W. Lee Y. Kim and Y. S. Myung, arXiv:0907.3568 [hep-th].

[11] J. Z. Tang and B. Chen, Phys. Rev. D 81043515 (2010).

[12] E. Kiritsis and G. Kofinas, arXiv:0910.5487 [hep-th].

[13] T. Harada, U. Miyamoto and N. Tsukamoto, Int. J. Mod. Phys. D 20 111(2011).

[14] E. Kiritsis, Phys. Rev. D 81 044009(2010).

[15] D. Capasso and A. Polychronakos, arXiv:0911.1535v3 [hep-th].

[16] J. Tang, arXiv:0911.3849[hp-th].

[17] H. W. Lee, Y. Kim, Y. S. Myung, Eur. Phys. J. C 70 367(2010).

[18] G. Koutsoumbas and P. Pasipoularides, arXiv:1006.3199v3 [hep-th].

[19] J. Greenwald, A. Papazoglou and A. Wang, Phys. Rev. D 81 084046(2010).

[20] R. G. Cai and A. Wang, Phys. Lett. B 686 166(2010).

[21] G. Koutsoumbas, E. Papantonopoulos, P. Pasipoularides and M. Tsoukalas, arXiv:1004.2289v2 [hep-th].

[22] A. N. Aliev and C. Senturk, Phys. Rev. D. 82 104016(2010).

[23] E. Gruss, arXiv:1005.1353v1 [hep-th].

[24] A. Kehagias and K. Sfetsos, Phys. Lett. B 678(1) (2009) 123.

[25] R. G. Cai, ,L. M. Cao and N. Ohta, Phys. Lett. B 679 504(2009).

[26] Y. S. Myung, arXiv:0905.0957v2 [hep-th].

[27] S. Chen, J. Jing, Phys. Rev. D 80 024036(2009).

[28] Y. S. Myuang, Phys. Rev. D 81 064006(2010).

[29] J. J. Peng and S. Q. Wu, Eur. Phys. J. C 66 325(2010).

[30] L. Iorio and M. L. Ruggiero, Int. J. Mod. Phys. A 25 5399(2010).

[31] L. Iorio and M. L. Ruggiero, Open Astron. J. 3167(2010).

[32] T. Harko, Z. Kovacs and F. S. N. Lobo, Proc. R. Soc. A467 1390(2011).

[33] T. Harko, Z. Kovacs and F. S. N. Lobo, Phys. Rev. D 80 044021(2009).

[34] M. R. Setare and M. Jamil, Int. J. Theor. Phys. 50 511(2011).

[35] W. Janke, D. A. Johnston and R. Kenna, arXiv:1005.3392v2 [hep-th].

[36] B. Gwak and B. H. Lee, JCAP 09 031(2010). 
[37] M. R. Setare and D. Momeni, Int. J. Theor. Phys. 49 106(2011).

[38] C. Ding. S. Chen and J. Jing, Phys. Rev. D 81 024028(2009).

[39] B. R. Majhi, Phys. Lett. B 686 49(2010).

[40] S. Chen and J. Jing, Phys. Lett. B 687 124(2010).

[41] R. A. Konoplya, Phys. Lett. B 679 499(2009).

[42] C. Wang and Y. Gui, Astrophys and Space Sci 325, 85(2010).

[43] K. Lin, N. Yang and J. Li, Int. J. Theor. Phys 50 48(2010).

[44] N. Varghese and V C Kuriakose, arXiv:1010.0549v1[gr-qc].

[45] M. R. Setare and D. Momeni, Mod. Phys. Lett. A 26 151(2011).

[46] K. D. Kokkotas and B. G. Schmidt, Living Rev. Relativ. 2 2(1999). H. P. Nollert, Class. Quantum Grav. 16 R159(1999)

[47] R. H. Price Phys. Rev. D. 5 2419(1972).

[48] S. Hod and T. Piran Phys. Rev. D. 58 044018(1998).

[49] L. E. Simone and C. M. Will, Class. Quantum Qrav. 9 963(1992), R. A. Konoplya, Phys. Lett. $B 550117(2002)$.

[50] K. Koyama and A. Tomimatsu, Phys. Rev. D. 63 064032(2001).

[51] K. Koyama and A. Tomimatsu, Phys. Rev. D. 64 044014(2001).

[52] R. Moderski and M. Rogatko, Phys. Rev. D. 64 044024(2001).

[53] R. A. Konoplya and A. Zhidenko Phys. Rev. D. 75 084004(2007).

[54] L. M. Burko and G. Khanna, Phys. Rev. D. 70 044018(2004).

[55] J. L. Jing, Phys. Rev. D. 70065004 (2004).

[56] D. Birmingham, I. Sachs and S. N. Solodukhin Phys. Rev. Lett. 88151301 (2002).

[57] E. Abdalla, B. Wang, A. L. Santos, W. G. Qiu, Phys. Lett. B 538435 (2002).

[58] L. Xue and B. Wang, Phys. Rev. D. 66024032 (2002).

[59] C. Gundlach, R. H. Price, and J. Pullin, Phys. Rev. D 49, 883 (1994).

[60] R. A. Konoplya and A. Zhidenko, Phys. Rev. D. 77 104004(2008).

[61] R. H. Price, Phys. Rev. D 5, 2419(1972), R. H. Price, Phys. Rev. D 5, 2439(1972).

[62] B. F. Schutz and C. M. Will, Astropphys. J. 291 (1985) L33-6.

[63] S. Iyer and C. M. Will, Phys. Rev. D. 35 (1987) 3621. 